\title{
Neutron Flux Intercomparison at NBS
}

\author{
Kenneth C. Duvall, Michael M. Meier, and Oren A. Wasson \\ Center for Radiation Research, National Bureau of Standards, Washington, DC 20234 \\ Vinh D. Huynh \\ Bureau Internaltional des Poids et Mesures, Sevres, France
}

\begin{abstract}
August 8, 1978
National Bureau of Standards (NBS) participation in an International Bureau of Weights and Measures (BIPM) sponsored neutron flux intercomparison is described. The efficiencies of two transfer instruments, a gasfilled ${ }^{3} \mathrm{He}$ proportional counter and a $\mathrm{BF}_{3}$ counter imbedded in a polyethylene sphere, were determined at neutron energies of $250 \mathrm{keV}$ and $560 \mathrm{keV}$. The efficiency of the ${ }^{3} \mathrm{He}$ detector was determined by placing it in the flux monitored by the NBS "black" detector. Since the polyethylene sphere detector shadows the "black" detector, a secondary $\mathrm{BF}_{3}$ counter was calibrated and it normalized the sphere and "black" detector runs. The efficiencies are reported and compared to the quantities determined at other participating standards laboratories.
\end{abstract}

Key words: Calibration standards; efficiency; international comparisons; neutron detectors; neutron flux.

\section{Introduction}

The International Bureau of Weights and Measures (BIPM) is sponsoring an intercomparison of neutron flux monitoring at the major international standards laboratories. NBS participation occurred during the period December 1 to 15,1976 at the neutron flux facility associated with the 3 MV positive ion Van de Graaff. Flux monitoring at this facility is done with a "black" neutron detector which has been calibrated through the associated particle technique and calculated using a Monte Carlo program. The NBS flux was intercompared at neutron energies of 250 and $565 \mathrm{keV}$. For these energies the intercomparison monitors used in the program are a ${ }^{3} \mathrm{He}$ gas filled proportional counter and a $\mathrm{BF}_{3}$ counter imbedded in a polyethylene sphere.

All other standards laboratories use an open geometry for measurements and the intercomparison is usually performed with the BIPM intercomparison monitor and the laboratory flux monitor symmetrically located equidistant from the neutron source. Since the NBS system employs a single beam, collimated and monitored at $0^{\circ}$, use of this technique was not possible. Instead, the "black" detector was used to calibrate a secondary monitor at a back angle and the latter was used to normalize intercomparison monitor and "black" detector runs.

Backgrounds for the "black" detector were measured using pulsed beam techniques. Since the transfer instruments were too slow for time-of-flight (TOF) measurements, backgrounds were determined in other ways. The sphere background was determined by studying the radial dependence of the count rate and by interposing a shadow bar between source and detector. Since the ${ }^{3} \mathrm{He}$ detector is a spectrometer, a curve fitting procedure could be used to discriminate against background.

The objective of the procedure was to measure an efficiency for each of the two intercomparison instruments at the two selected energies. Since measurements at other laboratories made use of the symmetry mentioned above, this efficiency was defined to include the detector geometry and no correction was made for air transmission between the neutron source and detector.

\section{Experimental}

\subsection{Neutron Source}

The source of neutrons was the ${ }^{7} \mathrm{Li}(p, n)$ reaction. Metallic lithium was vacuum evaporated onto a tantalum substrate that formed the end of the beam transport system. The targets were transported from the evaporator to the beamline in a helium atmosphere and care was taken to minimize their exposure to air. No evidence for oxidation of surface layers was seen in the threshold measurements. The target thickness was measured to be $17 \mathrm{keV}$ by observing the yield from the target as a function of bombarding energy near threshold and by the width of the neutron peak observed in the black detector TOF spectrum. The target spot was defined by two collimators in the proton beam line which were also used in the neutron collimator alignment. The 
discolored region where the beam struck the target indicated that the effective spot size was less than $4 \mathrm{~mm}$. Target current and currents incident on each collimator were monitored throughout the experiment.

To reduce room scattered neutrons the source was surrounded with a massive shield of paraffin loaded with $\mathrm{Li}_{2} \mathrm{CO}_{3}$. Machined inserts of lithium loaded polyethylene defined the neutron beam. These were aligned, along with the shield, using the same transit that defined the proton beam and the neutron collimator axis. Alignment was facilitated by use of an air bearing that supported the shield and a set of four screws rigidly attached to the floor which located the air bearing.

\subsection{Transfer Instruments}

Signals from the sphere detector were preamplified, amplified, and supplied to a single channel analyzer (SCA) operated in the lower level mode. The logic output of the SCA was scaled. The discriminator level of the SCA was adjusted to give a selected rate when a calibrated source was positioned in a reproducible location in the sphere. This calibration procedure was employed on a daily basis to eliminate possible shifts in the bias level.

The ${ }^{3} \mathrm{He}$ detector used a similar amplifier-SCA arrangement, but the amplifier output was also supplied to a multichannel analyzer (MCA). Pulse height spectra were recorded and output for subsequent analysis.

The collimated neutron beam depicted in figure 1 has a half angle of $4.5^{\circ}$. A line was scribed on the Al floorplate below and parallel to the beam axis so that by using a plumb bob it was easy to locate the sphere detector on the axis to within $\pm 2 \mathrm{~mm}$. Several measurements with the sphere

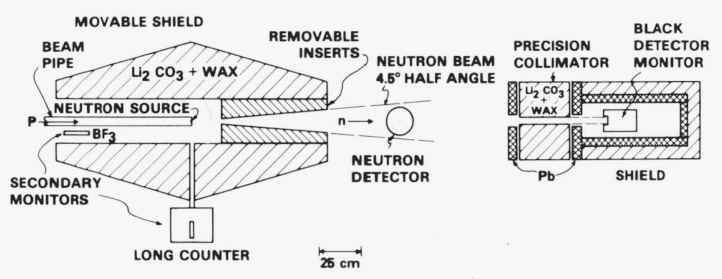

FIGURE 1. NBS neutron flux facility.

displaced from the axis by as much as $0.5 \mathrm{~cm}$ were done at a distance of $150 \mathrm{~cm}$. As expected from earlier beam profile measurements, no change in response was observed. At distances closer to the target it was possible to view the target spot from behind the sphere to insure that the entire detector was in the direct flux.

The ${ }^{3} \mathrm{He}$ detector was displaced $2.8^{\circ}$ from the neutron beam axis so that the black detector could be operated simultaneously with no transmission correction. Earlier profile measurements indicate that the beam uniformity is such that no flux correction need be made for such a displacement. The source-detector distance was $105 \mathrm{~cm}$.

\subsection{Black Detector}

The NE-110 black detector [1] $]^{1}$ was located in a massively shielded enclosure $5.86 \mathrm{~m}$ from the target. A $15.5 \mathrm{~cm}^{2}$ collimator defines the solid angle subtended by the detector and insures that all the neutron flux is incident on the reentrant hole of the detector. A transit was used to align the collimator with the neutron source. The last two proton beam collimators and the neutron collimator axis were aligned to be colinear to within $0.5 \mathrm{~mm}$. The effective area of the cylindrical collimator was increased from the geometrical area to account for the divergent neutron beam. This correction was one percent.

The electronics for the black detector are shown in figure 2. The system collects data from the black detector in a 4096 channel two-parameter array; pulse height and neutron timeof-flight. The ADC's are gated by true time-of-flight events to keep dead-time associated with photomultiplier noise to a minimum.

A loss of efficiency in monitoring may occur if a neutron event is not accompanied with a stop pulse. To measure the effect, a time-to-amplitude converter (TAC) with a $2 \mu \mathrm{s}$

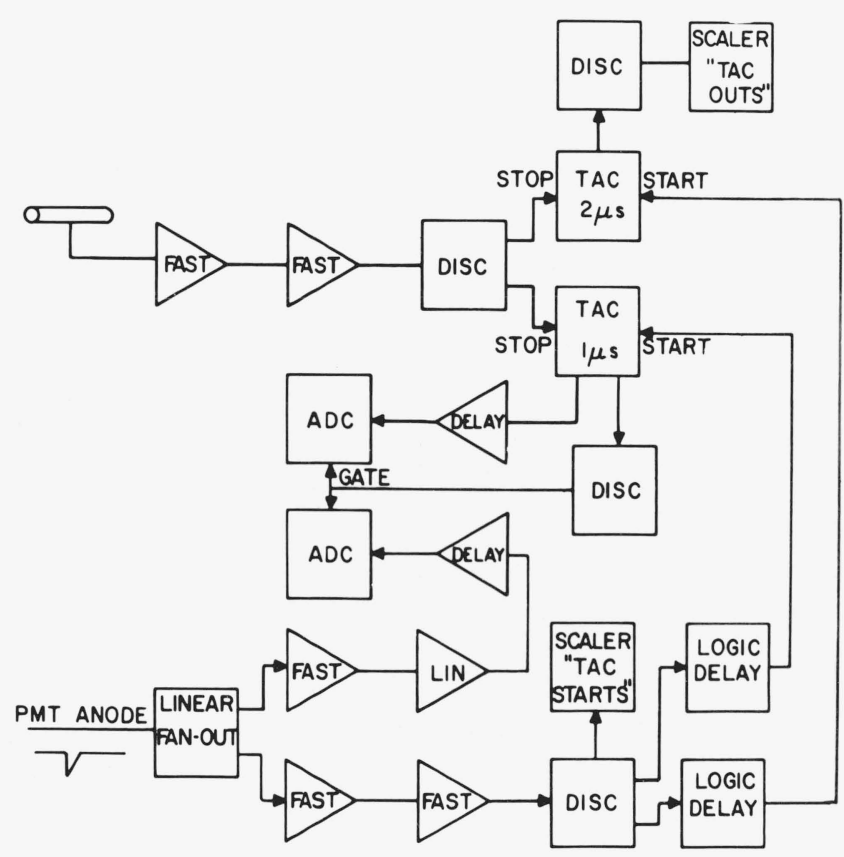

Figure 2. Two-parameter data acquisition system for the black detector.

\footnotetext{
${ }^{1}$ Figures in brackets indicate the literature references at the end of this paper.
} 
range was used and valid outputs were scaled and compared to the total number of TAC starts. Discrepancies between these two numbers were always less than 0.1 percent and the data was left uncorrected for this effect. The bias was set well into the photomultiplier tube (PMT) noise for the measurement at $250 \mathrm{keV}$ and it was not possible to make this check due to multiple firing of the discriminator.

The ADC's were read by a special unit [2] which converted the two outputs into a single address in a 32 (time) $\times 128$ (pulse-height) array. This was done so that address generation could be done by hardware, rather than the relatively time-consuming software, thereby reducing dead-time. This address was acquired by the computer through CAMAC and the approprite location in memory was incremented. A separate ADC was used to record the time-of-flight spectrum in the computer. The data acquisition program was triggered at the highest available priority and dead-time was calculated and incremented in the course of each program execution.

\subsection{Secondary Monitors}

Since the black detector could not be used with the polyethylene sphere shadowing the beam, several secondary monitors were used to normalize sphere and black detector runs. The shield surrounding the source has a $2.54 \mathrm{~cm}$ diameter hole which views the target at an angle of $90^{\circ}$. A long counter (LC) mounted outside this hole was tested for use as a suitable secondary monitor. By steering the beam transversely across a distance of less than $4 \mathrm{~mm}$ at the target it was found that the rate in the $\mathrm{LC}$ varied $\sim 10$ percent. Also when the target was rotated by $30^{\circ}$ to produce a new beam spot, the rate changed by as much as 25 percent. These effects were attributed to the transmission of neutrons through the tantalum substrate. These large changes made the long counter unacceptable as a monitor.

A second monitor was a plastic scintillator which was biased above tube noise and located at $180^{\circ}$ to the proton beam and about $1.0 \mathrm{~m}$ from the target. This monitor exhibited sporadic behavior which may have been associated with sensitivity to gamma rays from collimating slits, shifts in the bias level and the fact that the neutron energies at $180^{\circ}$ are $140 \mathrm{keV}$ and $15 \mathrm{keV}$ for $0^{\circ}$ energies $565 \mathrm{keV}$ and $250 \mathrm{keV}$ respectively. This monitor was also rejected.

Finally, a $\mathrm{BF}_{3}$ proportional counter was mounted on the beam pipe about $25 \mathrm{~cm}$ behind the target. Figure 3 shows the ratio of the rate of this detector to the black detector for an eighteeen hour period during the $250 \mathrm{keV}$ sphere runs. The excellent stability of the $\mathrm{BF}_{3}$ counter made it the obvious choice for a secondary monitor.

\section{Data Collection}

The procedure followed for determining the sphere efficiency consisted of calibrating the $\mathrm{BF}_{3}$ counter relative to the

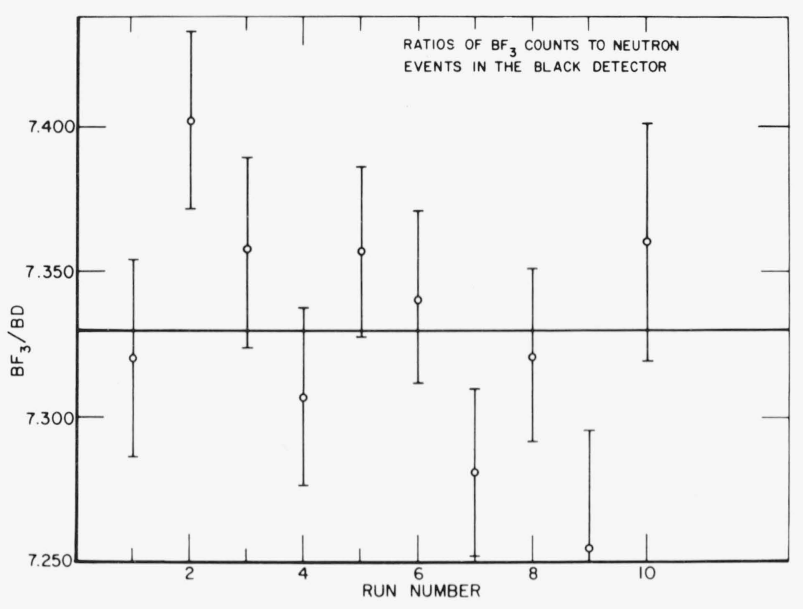

FIGURE 3. Ratio of black detector to secondary monitor counting rates. Error bars are based on counting statistics $(1 \sigma)$ for the detectors.

black detector and then taking data with the sphere detector relative to $\mathrm{BF}_{3}$.

The ordinary sequence of runs was to alternate calibration runs with sphere runs. At frequent intervals the beamstop was used to shield the target and prevent neutron production and black detector data was taken. This provided a "beamstop-in" background, comprised of photomultiplier noise, cosmic rays, and other events not related to neutron production at the target. For the calibration runs the two parameter pulse-height and time-of-flight spectrum as well as a single parameter time-of-flight spectrum were recorded on magnetic tape. Also recorded were computer dead-time, total integrated current, average target current, time and scaler outputs. Included in the latter were sphere events, $\mathrm{BF}_{3}$ events, black detector starts, and black detector TAC conversions as discussed above. Sums over the pulse height spectra for each of 22 time channels in the two parameter array were also recorded. These sums permitted preliminary calculations of calibration constants as well as the influence of various background contamination. It was also possible to observe the stability of the monitors and of the pulsing system with this information.

Measurements were then made with the sphere in the beam and the above data were taken except those from the black detector since it was shadowed by the sphere. The sphere to target distance was $1.5 \mathrm{~m}$ for the intercomparison runs and varied between 1.4 and $3.0 \mathrm{~m}$ for the background determination runs. A run with a massive shadow bar interposed between source and sphere constituted a second method for background determination.

Since the ${ }^{3} \mathrm{He}$ counter is small it was possible to do calibration runs simultaneously with the data collection. The ${ }^{3} \mathrm{He}$ data were comprised of 256 channel pulse height spectra and analyzer dead-time. These were output for later analysis. The spectral sum above a given bias served for preliminary analysis. 


\section{Data Analysis}

The count rate in the black detector is

$$
R_{B D}=\varphi \frac{A_{\mathrm{col}}}{\left(r_{\mathrm{col}}\right)^{2}} \epsilon T\left(r_{B D}\right)
$$

where

$R_{B D}$ is the background and dead-time corrected rate in the black detector

$\varphi$ is the neutron source strength

$A_{\text {col }}$ is the area of the neutron collimator

$r_{\text {col }}$ is the target-neutron collimator distance

$T(r)$ is air transmission as a function of the distance $r$

$r_{B D}$ is the target-black detector distance

$\boldsymbol{\epsilon}$ is the black detector efficiency

For the rate in a transfer counter, $R_{c}(r)$

$$
R_{c}(r)=\varphi \frac{A_{\mathrm{eff}}}{r^{2}} E^{\prime} T(r)
$$

where

$A_{\text {eff }}$ is the effective area of the counter

$r$ is the target-counter distance

$E^{\prime}$ is the intrinsic efficiency of the counter

We may define an efficiency* which takes into account the geometric effects:

$$
E \equiv \frac{A_{\mathrm{eff}}}{r_{c}{ }^{2}} E^{\prime}
$$

where $r_{c}$ is the conventional distance at which measurements are made.

Then

$$
R_{c}(r)=\varphi\left(\frac{r_{c}}{r}\right)^{2} T(r) E
$$

Since

$$
R_{m}=k \varphi
$$

where $R_{m}$ is a monitor rate, we can obtain

$$
\frac{R_{B D}}{R_{m}(B D)} * \frac{R_{m}(c)}{R_{c}(r)}=\frac{A_{\mathrm{col}}}{R_{\mathrm{col}^{2}}} \frac{T\left(r_{B D}\right)}{T(r)} \frac{\epsilon}{E}\left(\frac{r}{r_{c}}\right)^{2}
$$

Finally

$$
E=\left(\frac{R_{c}(r)}{R_{m}(c)}\right)\left(\frac{R_{B D}}{R_{m}(B D)}\right)^{-1} \frac{A_{\mathrm{col}}}{r_{\mathrm{col}}^{2}}\left(\frac{r}{r_{c}}\right)^{2} T\left(r_{B D}-r\right) \epsilon
$$

where $R_{m}(c)$ is the monitor rate with the counter in position.

* The efficiency, $E$, is referred to as the "sensitivity of the transfer instrument, $\boldsymbol{\epsilon}_{\mathbf{\Omega}}$ " in ref. [4].
The corrections made to quantities in the above formula and associated uncertainties are listed in table I.

TABLE I. Corrections and Uncertainties

\begin{tabular}{l|c|c}
\hline \hline \multicolumn{1}{c|}{ Correction } & Magnitude & Uncertainty \\
\hline $\begin{array}{l}\text { Black detector summation integral am- } \\
\text { biguity }\end{array}$ & - & $0.4 \%$ \\
$\mathrm{BF}_{3}$ background & $<0.01 \%$ & $<0.01$ \\
Sphere background & 1.0 & 1.0 \\
Sphere deadtime & $<0.01$ & $<0.01$ \\
${ }^{3}$ He uncertainty & - & 0.5 \\
Target collimator distance & - & 0.07 \\
Target counter distance & - & 0.4 \\
Air transmission & - & 0.4 \\
Black detector efficiency & - & 2.5
\end{tabular}

$250 \mathrm{keV}$

\begin{tabular}{l|l|l} 
Collimator area & $2.8 \%$ & $0.5 \%$ \\
Black detector background & 9.0 & 0.8 \\
Black detector gamma ray contamina- & 1.0 & 0.5 \\
$\quad$ tion of neutrons & & \\
Black detector deadtime & 0.8 & $<0.01$ \\
Black detector counting statistics $(1 \sigma)$ & - & 0.2 (Sphere) \\
& - & $0.1\left({ }^{3} \mathrm{He}\right)$ \\
$\mathrm{BF}_{3}$ counting statistics $(1 \sigma)$ & - & $0.14(\mathrm{Sphere})$ \\
& - & $0.07\left({ }^{3} \mathrm{He}\right)$ \\
Sphere counting statistics $(1 \sigma)^{3} \mathrm{He}$ counting statistics $(1 \sigma)$ & - & 0.8 \\
& - & 0.8
\end{tabular}

$560 \mathrm{keV}$

Collimator area

Black detector background

Black detector deadtime

Black detector counting statistics ( $1 \sigma)$

$\mathrm{Bf}_{3}$ counting statistics $(\mathrm{l} \sigma)$

Sphere counting statistics $(1 \sigma)$

${ }^{3} \mathrm{He}$ counting statistics $(1 \sigma)$

\begin{tabular}{l|l}
$.6 \%$ & $0.5 \%$ \\
.0 & 0.4 \\
.0 & $<0.01$ \\
- & 0.3 (Sphere) \\
- & $0.1\left({ }^{3} \mathrm{He}\right)$ \\
- & 0.2 (Sphere) \\
- & $0.1\left({ }^{3} \mathrm{He}\right)$ \\
- & 0.6 \\
- & 1.0
\end{tabular}

The monitor rate from the $\mathrm{BF}_{3}$ counter and counter rate from the sphere were taken from the scalers associated with each and corrected for background measured with the beamstop in. Since the $\mathrm{BF}_{3}$ counter served as a monitor, the $\sim 0.1$ percent dead-time correction cancels in first order and was therefore ignored. The rate in the ${ }^{3} \mathrm{He}$ counter was obtained by summing the counts above a given pulse height level. This level was taken to be 0.6 of the pulse height difference between the thermal and fast neutron peak. This analysis was done with a fitting program designed and written for the intercomparison at BIPM [3]. The cylindrical collimator area was calculated from its measured diameter and corrected for transmission due to the beam divergence. Collimator, detector, and black detector distances were all measured with commercial scales. Transmissions through air were calculated using distances measured as above and total cross 
sections from ENDF-B [4] files. Cross sections were assigned an uncertainty of 4 percent to account for a combination of inherent cross section uncertainty and possible energy shifts.

A typical black detector time-of-flight spectrum for 250 $\mathrm{keV}$ is schematically shown in figure 4 . Time regions corresponding to neutrons (A), background (B), and gamma rays $(\mathrm{C})$ are labeled. The time channels in each region were

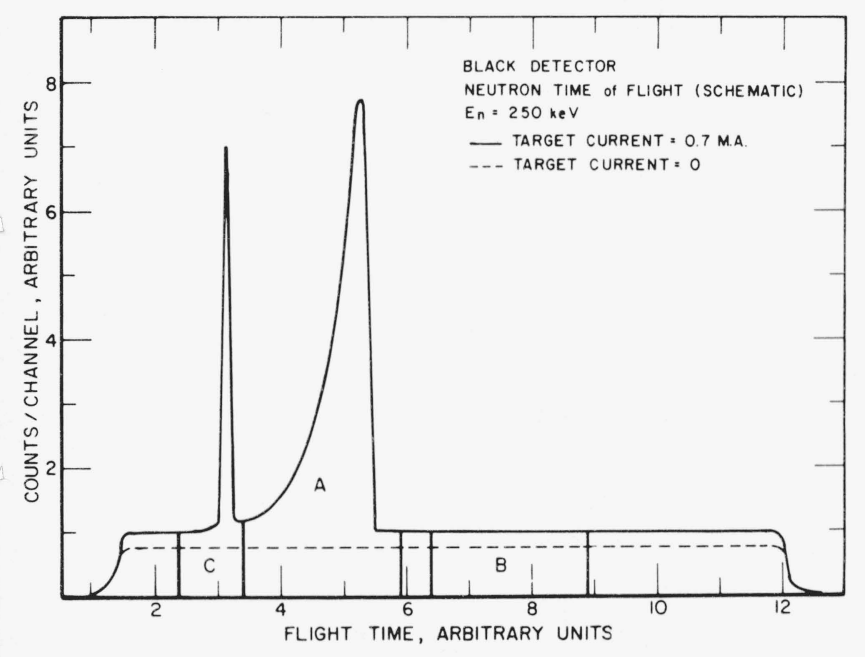

Figure 4. Time-of-flight spectrum for $250 \mathrm{keV}$ neutrons.

summed to produce pulse height spectra. The same operation was done for the two parameter spectrum taken with beamstop in to produce pulse height spectra for background. The latter was normalized and subtracted from the calibration spectrum and were found to account for roughly 80 percent of the background in region B. The remaining 20 percent background was due to neutron and gamma ray production in the target and showed no apparent structure in the reduced time spectrum. It was appropriately normalized and subtracted from the A and C spectra. A typical reduced C spectrum is shown in figure 5. The object of this part of the analysis was to determine the number of neutrons which were in the gamma ray time region. Since the gamma rays have relatively large light output, it was assumed that their pulse height distribution would be relatively flat in the vicinity of the $250 \mathrm{keV}$ neutrons. This seems to be born out by the experimental spectrum and therefore a straight line with zero slope is fit to the high pulse height events and extrapolated to zero. The remaining events above the gamma ray were then summed and subsequently used to correct the data. It was possible to repeat the analysis, expanding the $\mathrm{C}$ region to successively shorter times and finding a limiting value for this correction.

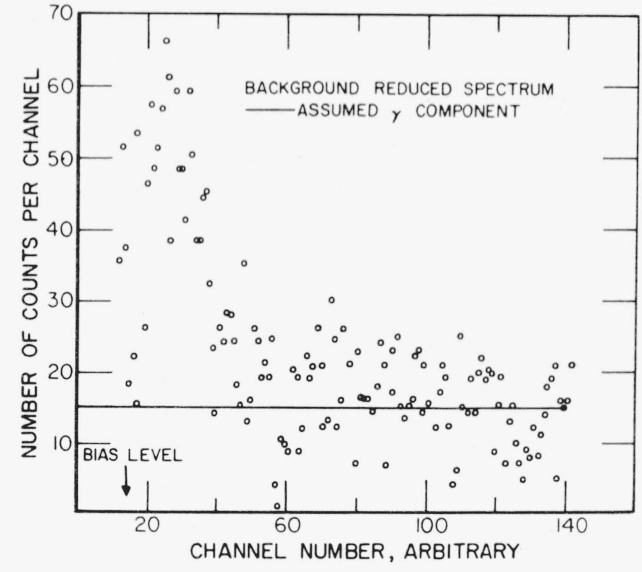

Figure 5. Pulse height spectrum for the time region of gamma rays.

Uncertainty in the background was obtained by ignoring the beam off background and making a direct subtraction of the $\mathrm{B}$ region from $\mathrm{A}$. Changing the summation limits from 5 to 10 time channels for each region was also helpful in assessing this uncertainty.

The channel width of the reduced A spectrum was then changed to give a spectrum which could be compared with the output of CARLY, [5] the Monte Carlo program for efficiency calculation of the black detector. Usually less than three iterations of channel width changes were sufficient to produce a qualitatively good fit. Figure 6 shows a reduced spectrum at $250 \mathrm{keV}$ and the corresponding CARLY calculation. The value for the rate in the black detector is taken to

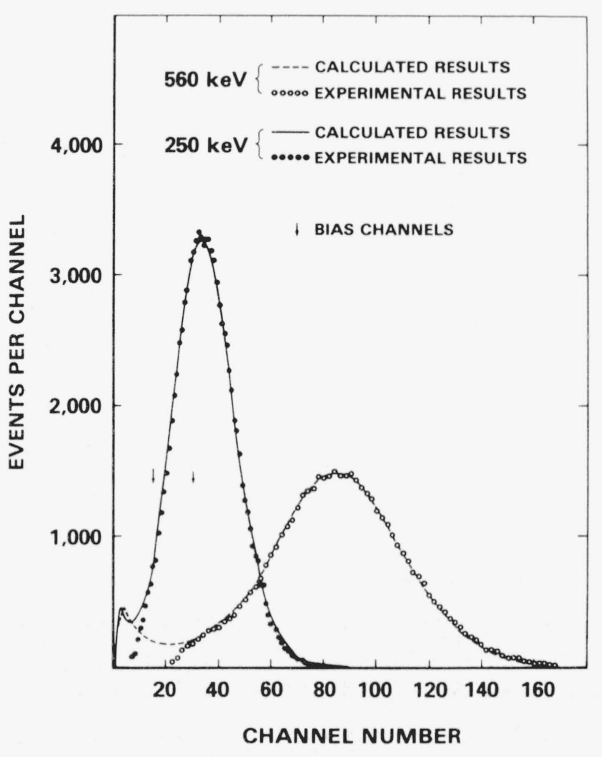

Figure 6. Experimental and Monte Carlo calculated pulse height spectra. 
be the sum of counts above the indicated bias level. The corresponding efficiency is deduced from the CARLY calculation using the same bias level. It was found that the quality of the fit deteriorated appreciably when the bin width parameter was adjusted away from optimum by an amount that shifted the peak by one channel. This criterion was used in assigning an uncertainty due to bias channel ambiguity between experiment and calculation.

A schematic representation of the time-of-flight spectrum for $560 \mathrm{keV}$ neutrons is shown in figure 7. Gamma rays do not complicate the analysis in this case, but additional neutrons, due to incompletely suppressed retrace pulses, appear in region $\mathrm{C}$. The analysis proceeded as above except that neutrons from region $\mathrm{C}$ were included as part of the main peak sum. A reduced spectrum and CARLY calculation are also shown in figure 6. Finally, computer data were corrected for dead-time, and efficiencies were calculated from the above formula.

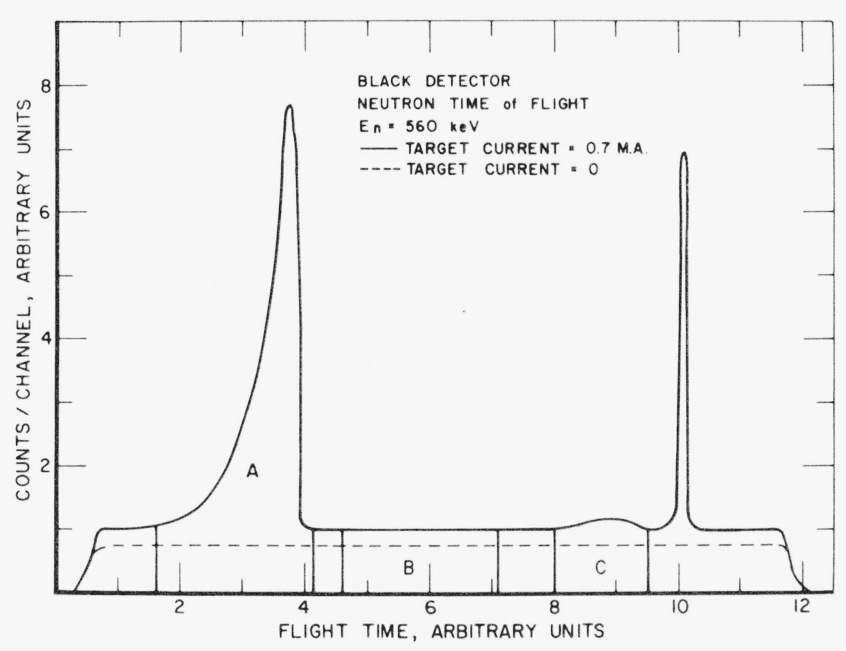

Figure 7. Time-of-flight spectrum for $560 \mathrm{keV}$ neutrons.

\section{Results}

The efficiency determination of sphere and ${ }^{3} \mathrm{He}$ counters is summarized in table II. The uncertainty associated with the measurement is derived from table $I$ by adding all applicable uncertainties in quadrature.

The $250 \mathrm{keV}$ results are graphically depicted in figure 8 along with the results obtained by other standards laboratories [3]. The ${ }^{3} \mathrm{He}$ results are in fairly good agreement as are the results for both detectors at $560 \mathrm{keV}$ as discussed in reference [3]. It is interesting to note that the discrepancies between laboratories for the sphere calibration are correlated with the background levels at each laboratory. The four

\begin{tabular}{cc|c|c}
\multicolumn{4}{c}{ TABle II. Efficiency $\left(\right.$ cts $\left./ n-s r^{-1}\right)$} \\
\hline \multirow{2}{*}{$E_{n}$} & $\begin{array}{c}\text { Net Statistical } \\
\text { Uncertainty }\end{array}$ & $\begin{array}{c}\text { Net Systematic } \\
\text { Uncertainty } \\
\text { Sphere }\end{array}$ & Efficiency \\
\hline $250 \mathrm{keV}$ & $0.8 \%$ & $3.0 \%$ & $4.76 \pm 0.15 \times 10^{-6}$ \\
$565 \mathrm{keV}$ & 0.7 & 2.9 & $5.97 \pm 0.18 \times 10^{-6}$ \\
& & & \\
& & & \\
& & & \\
& & & \\
$250 \mathrm{keV}$ & & $2.9 \%$ & $3.20 \pm 0.09 \times 10^{-6}$ \\
$565 \mathrm{keV}$ & 1.0 & 2.7 & $2.21 \pm 0.06 \times 10^{-6}$
\end{tabular}

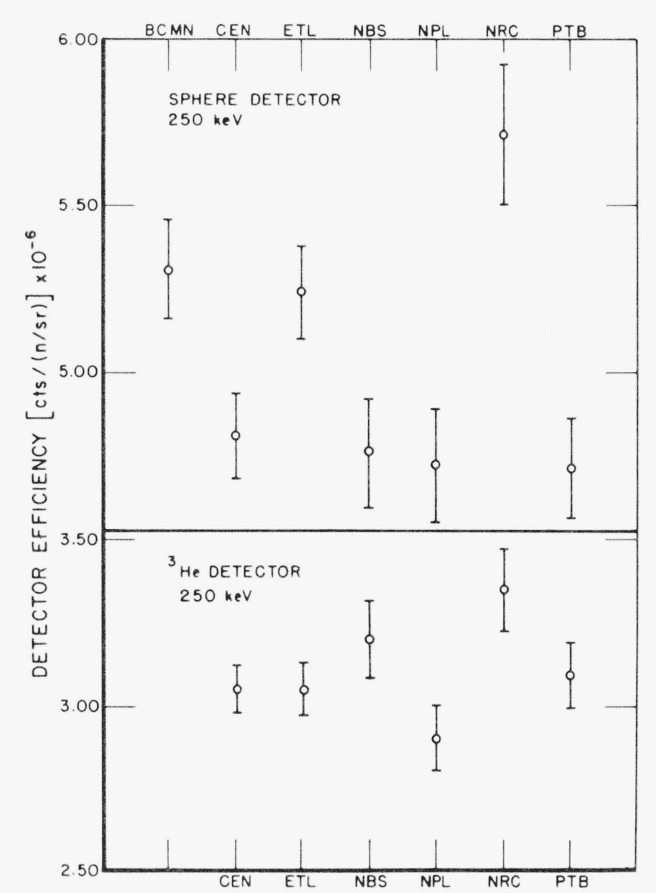

FiguRE 8. Detector efficiency determined at $250 \mathrm{keV}$ by NBS and other standards laboratories. BCMN - Bureau Central des Mesures Nucleaires, Geel, Belgium; CEN = Centre d'Etudes Nucleaires, Cadarache, France; ETL = Electrotechnical Laboratory, Tokyo, Japan; NBS = National Bureau of Standards, Washington, DC, U.S.A.; NPL = National Physical Laboratory, Teddington, Great Britain; NRC $=$ National Research Council, Ottawa, Canada; PTB $=$ Physikalisch-Technische Bundesanstalt, Braunschweig, Germany.

laboratories with the sphere efficiency of $\sim 4.70$ also reported the lowest backgrounds. These effects are much less pronounced for the ${ }^{3} \mathrm{He}$ detector which has some background discrimination and for the $560 \mathrm{keV}$ energy where the ${ }^{7} \mathrm{Li}(p$, $n$ ) source reaction is five times as prolific and the signal to background ratio is proportionately improved.

A second part of the measurement was an attempt to measure sphere background by varying the source-sphere distance. If one assumes an isotropic background resulting 
from wall return and air scatter, then the neutron distribution should be of the form

$$
N(r)=\frac{A}{r^{2}}+B,
$$

where $r$ is the source-sphere distance. The apparent efficiency of the detector will then increase as $r$ increases and the magnitude of the background can be inferred from the magnitude of this effect. Figure 9 is a plot of the efficiency values obtained for this radial variation. It is clear that systematic uncertainties shift the data beyond the limits expected from the statistical error bars. However, no systematic rise with increasing $r$ is observed and the contribution of an isotropic background is less than three or four percent at $1.5 \mathrm{~m}$. Another run with a massive shield designed to shadow the sphere showed no significant background above room background.

Neither result is unexpected. A shadow bar measurement is normally used to determine isotropic backgrounds in an open geometry. For the shielded geometry employed at NBS the shadow bar measurement could as well have been considered a "plugged" source run. Varying the distance, $r$, from source to detector is also conventionally applied to open geometry. For a shielded source the background would be expected not to be isotropic, but rather to originate in the components of the shield. This is a strong argument for using pulsed beam techniques and fast transfer instruments in future intercomparisons.

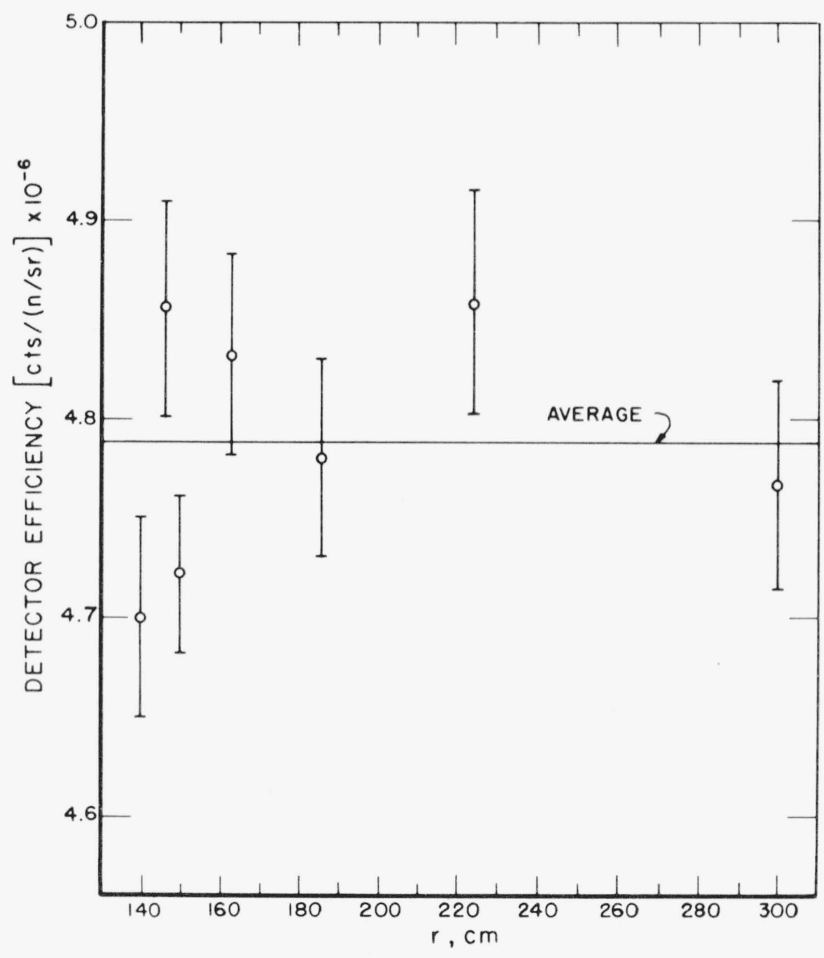

FiguRE 9. Sphere efficiency as a function of distance from the source.

No departure from $r^{-2}$ behavior is evident.

\section{References}

[1] Lamaze, G. P., Meier, M. M., and Wasson, O. A., Conf. on Nuclear Cross Sections and Technology, Nat. Bur. Stand. (U.S.), Spec. Publ. 425, Vol. 1, pp. 73-74 (Oct. 1975).

[2] Green, David, Private communication.
[3] Huynh, V. D., International Specialists' Symp. on Neutron Standards and Applications, Nat. Bur. Stand. (U.S.), Spec. Publ. 493, p. 244, (Oct. 1977).

[4] Evaluated Nuclear Data File, Format B, Version IV, National Nuclear Data Center, Brookhaven National Laboratory, Upton, N.Y.

[5] M. M. Meier, Private communication. 